Conclusion Longitudinal myocardial strain, as revealed by strain-encoded CMR, is associated with chronological age in healthy adults.

Funding Medical Research Scotland project grant. Professor Berry was supported by a Senior Clinical Fellowship from the Scottish Funding Council.

\section{PEAK CIRCUMFERENTIAL STRAIN ASSESSED USING STRAIN-ENCODED CARDIAC MAGNETIC RESONANCE (DENSE) IN HEALTHY VOLUNTEERS AT 1.5- AND 3.0 TESLA}

1,2Kenneth Mangion, ${ }^{1}$ Guillaume Clerfond, 1,2David Carrick, 'John McClure, ${ }^{1}$ Samuli Rauhalammi, ${ }^{1,2}$ David Corcoran, ${ }^{3}$ Christie McComb, ${ }^{1}$ Aleksandra Radjenovic, ${ }^{1,2}$ Colin Berry ${ }^{1}$ BHF Glasgow Cardiovascular Research Centre, University of Glasgow, UK; ${ }^{2}$ West of Scotland Heart and Lung Centre, Golden Jubilee National Hospital, Clydebank, UK; ${ }^{3}$ Clinical Physics, NHS Greater Glasgow and Clyde, Glasgow, UK

\subsection{6/heartjnl-2015-307845.24}

Background Displacement Encoding with Stimulated Echoes (DENSE) is a strain-encoded cardiac magnetic resonance (CMR) method that directly quantifies left ventricular (LV) mechanics within myocardial regions. Strain values might vary according to CMR field strength. In order to assess this possibility, we implemented strain-encoded CMR at 1.5 Tesla and 3.0 Tesla with DENSE in healthy adults.

Methods Healthy volunteers without any medical history or treatment gave informed consent and underwent CMR at $1.5 \mathrm{~T}$ (Magnetom Avanto, Siemens Healthcare) and 3.0T (Magnetom Verio; Siemens Healthcare) within $24 \mathrm{~h}$. The imaging protocol included balanced steady state free precession (b-SSFP) sequences for cardiac anatomy and function and strain-encoded CMR with DENSE. (CIM_DENSE2D software (University of Auckland, New Zealand; Siemens Healthcare). Global- and segmental circumferential strain was measured at the midventricular level.

Results Sixty five participants (31 (48\%) male, mean age $44 \pm$ 18) were included (Table 1). Mean global and regional strain values differed slightly between $1.5 \mathrm{~T}$ and $3.0 \mathrm{~T}$, and these differences were mainly related to strain values in the anterior and anterolateral LV segments were LV displacement is greatest.

Conclusion Strain-encoded CMR with DENSE provides regionally localised measurements of circumferential strain. Subtle field-strength differences were apparent for anterior/anterolateral LV strain.

Funding Medical Research Scotland project grant. Professor Berry was supported by a Senior Clinical Fellowship from the Scottish Funding Council.

Abstract 24 Table 1 Global Circumferential Strain in 65 Healthy Volunteers

\begin{tabular}{llll}
\hline Circumferential Strain (mid LV level) * & $1.5 \mathrm{~T}$ & 3.0 T & p-value \\
\hline Mean value & $19.62 \pm 2.6$ & $18.78 \pm 2.4$ & 0.011 \\
Anterior & $21.32 \pm 3.7$ & $20.00 \pm 3.5$ & 0.014 \\
Antero-septal & $18.39 \pm 3.5$ & $17.70 \pm 3.3$ & 0.200 \\
Infero-septal & $16.69 \pm 3.5$ & $15.87 \pm 3.5$ & 0.118 \\
Inferior & $19.98 \pm 3.5$ & $20.26 \pm 3.6$ & 0.580 \\
Infero-lateral & $21.84 \pm 3.4$ & $21.17 \pm 3.8$ & 0.165 \\
Antero-lateral & $21.66 \pm 3.5$ & $20.42 \pm 3.6$ & 0.006 \\
\hline *Expressed as mean, standard deviation & & &
\end{tabular}

\section{THE USE OF CARDIOVASCULAR MAGNETIC RESONANCE (CMR) IMAGING IN THE ASSESSMENT OF PERSONNEL IN THE UK ARMED FORCES}

${ }^{1}$ IT Parsons, ${ }^{2} \mathrm{R}$ Chamley, ${ }^{2,3} \mathrm{ED}$ Nicol, ${ }^{3,4} \mathrm{~J}$ d'Arcy. ${ }^{1}$ Royal Brompton Hospital, London, UK; ${ }^{2}$ St George's Hospital, London, UK; ${ }^{3}$ Clinical Aviation Medicine Service, RAF Henlow, Bedfordshire, UK; ${ }^{4}$ Oxford Centre for Clinical Magnetic Resonance Research, John Radcliffe Hospital, Oxford, UK

\subsection{6/heartinl-2015-307845.25}

Introduction The Armed Forces places considerable occupational demands on its workforce. Cardiovascular disease (CVD) remains a common cause of death and premature discharge. Simple CVD screening is performed periodically throughout the careers of service personnel, particularly in those in high risk roles such as aircrew. Abnormalities commonly require investigation with advanced cardiovascular imaging.

Methods A retrospective cohort analysis of all military CMR requests to two military cardiologists was undertaken to determine demographics, reason for referral, results of investigation and occupational outcome.

Results Between 2011 and 2015, fifty-six patients (95\% male, median age 44 years) were referred for CMR. The commonest indications were abnormal echocardiography (21\%), ventricular ectopy (16\%), suspicion of cardiomyopathy (13\%) and other asymptomatic ECG abnormalities ( $\mathrm{T}$ wave inversion (11\%) and bundle branch block (7\%)).

Of the CMR scans performed, 25\% demonstrated features confirming cardiomyopathy (25\% dilated and $12.5 \%$ hypertrophic phenotype). CMR features of athletic heart were seen in $11 \%$ of scans, and considered potentially suggestive of early cardiomyopathy. A quarter of patients had significant late gadolinium enhancement, in a variety of pathological patterns. Adenosine stress imaging was performed in $21 \%$ of patients, a quarter of which demonstrated perfusion abnormalities.

Whilst $30 \%$ of patients were able to return to unrestricted duties following CMR, 70\% required occupational restriction, mostly in aircrew. Of these $18 \%$ were unable to fly pending further review, 36\% were deemed unfit to fly solo, and $7 \%$ had restrictions placed on their ability to deploy on overseas operations.

Conclusion CMR is a valuable tool in the investigation of military personnel, and a useful adjunct to determine occupational, as well as clinical, risk. With the recent introduction of more rigorous CVD screening to join the Army, and the importance of comprehensive risk assessment in aircrew, the use of CMR in this population is likely to increase.

\section{CARDIOVASCULAR MAGNETIC RESONANCE ACTIVITY IN THE UNITED KINGDOM: RESULTS OF THE 2014 BSCMR SURVEY}

${ }^{1}$ DP Ripley, ${ }^{2} \mathrm{DM}$ Sado, ${ }^{3} \mathrm{GP}$ McCann, ${ }^{4} \mathrm{C}$ Berry. ${ }^{1}$ Multidisciplinary Cardiovascular Research Centre (MCRC) and Leeds Institute of Cardiovascular and Metabolic Medicine, University of Leeds, Leeds, UK; ${ }^{2}$ King's College London, Cardiovascular Division, London, UK; ${ }^{3}$ Department of Cardiovascular Sciences, University of Leicester, and the National Institute of Health Research Leicester Cardiovascular Biomedical Research Unit, Leicester, UK; ${ }^{4}$ BHF Glasgow Cardiovascular Research Centre, Institute of Cardiovascular and Medical Sciences, University of Glasgow, Glasgow, UK; On behalf of the British Society of Cardiovascular Magnetic Resonance

10.1136/heartjnl-2015-307845.26 
Conflicts: Nil

Background The 2011 British Society of Cardiovascular Magnetic Resonance (BSCMR) national survey demonstrated a marked year-on-year increase in the number of CMR scans per centre (2008-2010) and in the total number of CMR scans performed annually in the UK overall. ${ }^{1}$

Methods We undertook the second national survey on behalf of the BSCMR to identify all adult CMR providers within the UK in 2014. The questionnaire was sent to the lead clinician for CMR at each site and electronically reported.

Results Of the 68 adult CMR centres identified in the UK, $55(81 \%)$ responded to the questionnaire. Thirty three (60\%) were tertiary cardiac centres and $22(40 \%)$ were district general hospitals. Thirty six hospitals (65\%) had a level 3 accredited trainer on site with 559 trainees (87\% cardiology) at various levels of training. One hundred and twenty five trainees were at the level 3 stage of training ( $75 \%$ of which were from 5 centres). Thirty three hospitals $(60 \%)$ had a formal training program for cardiology and 17 (31\%) for radiology trainees. The majority of hospitals (44 (80\%)) had a quality control program in place.

For activity in 2013: 5 centres (9\%) provided no details on total numbers of scans, $9(16 \%)$ performed $<300$ scans, 14 (25\%) 300-500, 7 (13\%) 501-1000 and 20 (36\%) >1000 scans. The total number of reported scans increased by $253 \%$ between $2008(23,216)$ and $2013(58,936)$. Mean number of scans per centre are shown in Figure 1.

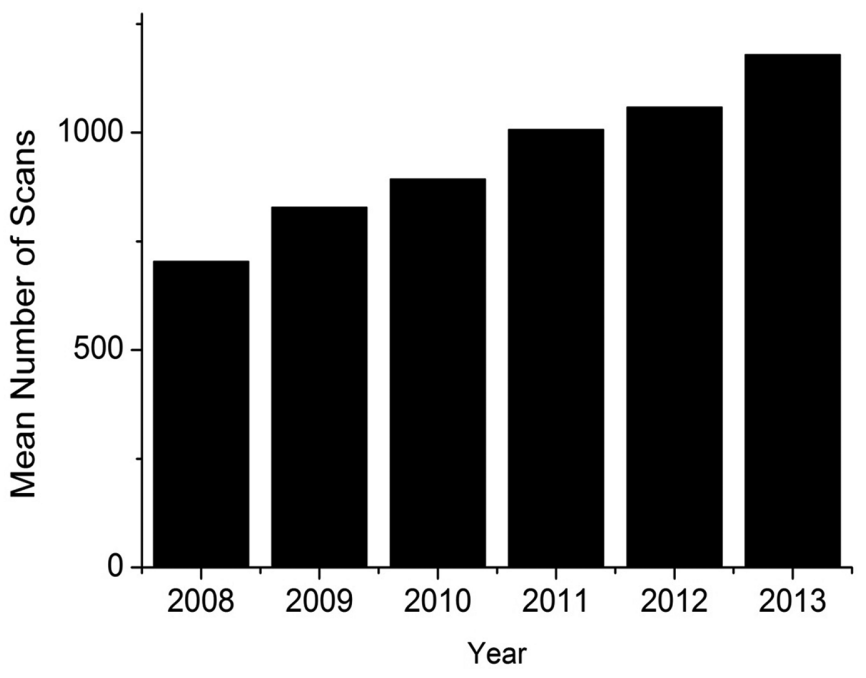

Abstract 26 Figure 1 Annual mean number of CMR scans reported in the 2014 British Society of Cardiovascular Magnetic Resonance Survey between years 2008-2013 inclusive (2008:704 scans (range 10-5500); 2009:828 (10-5600); 2010:893 (65-5700); 2011:1007 (35-6000); 2012:1058 (40-6200); 2013:1179 (48-6500))

Conclusion There is evidence of a sustained increase in CMR service provision in the UK. Most centres provide a CMR training program and quality assurance.

Acknowledgements We would like to thank Drs S. Nair, M. Motwani, V. Ferriera, E. McAlindon, N. Edwards, D. Carrick, M. Goonewardene, A. McDiarmid, A. Uddin, S. Kannoly and H. Douglas for their help with this audit.

\section{REFERENCE}

1 Anthony $R$ et al. JCMR 2011;13:57

\section{REGIONAL VARIATIONS IN MYOCARDIAL T1 RELAXATION TIMES IN HEALTHY ADULTS AT 1.5 AND 3.0 TESLA}

${ }^{1} \mathrm{~S}$ Rauhalammi, ${ }^{1,2} \mathrm{D}$ Carrick, ${ }^{1} \mathrm{~K}$ Mangion, ${ }^{1} \mathrm{G}$ Clerfond, ${ }^{1} \mathrm{P}$ Hall Barrientos, ${ }^{1} \mathrm{C}$ McComb, ${ }^{1} \mathrm{~A}$ Radjenovic, ${ }^{1,2} \mathrm{C}$ Berry. ${ }^{1} \mathrm{BHF}$ Glasgow Cardiovascular Research Centre, University of Glasgow, UK; ${ }^{2}$ Golden Jubilee National Hospital, UK

\subsection{6/heartjnl-2015-307845.27}

Background Limited information is available on regional differences in myocardial T1 relaxation times in healthy adults. We used cardiac magnetic resonance (CMR) imaging at two field strengths, 1.5 - and 3.0 Tesla (T), to assess regional distribution of $\mathrm{T} 1$, in milliseconds (ms), in myocardial regions-of-interest (ROIs) in healthy adults.

Methods Healthy adults without history of cardiovascular disease underwent CMR at $1.5 \mathrm{~T}$ (Avanto) and 3.0 T (Verio). T1 maps were acquired in three left ventricular (LV) short-axis slices, using an optimised modified Look-Locker inversion recovery (MOLLI) investigational prototype sequence (Siemens WIP 448). ROIs were delineated using the American Heart Association 16-segment model.

Results 80 consecutive volunteers (41 (51.3\%) male, mean age $44 \pm 18$ years) underwent scans $1.5 \pm 1.4$ days apart. Because of artefacts related to cardio-respiratory motion and susceptibility effects, $44(3.4 \%)$ segments imaged at $1.5 \mathrm{~T}$ and $71(5.5 \%)$ at 3.0 T were excluded, respectively, with a preponderance occurring at the distal LV. Mean T1 values at 1.5 T/3.0 Twere 946.5 $\pm 50.3 \mathrm{~ms} / 1152.0 \pm 54.9 \mathrm{~ms}$. At $1.5 \mathrm{~T}$ there were regional differences across 16 segments (Table 1); T1 values from myocardial septal ROIs $(956.7 \pm 44.8 \mathrm{~ms})$ were higher than non-septal ROIs $(941.8 \pm 52.0 \mathrm{~ms}, \mathrm{p}<0.001)$. T1 was lower in lateral ROIs (939.7 $\pm 54.1 \mathrm{~ms})$ vs. non-lateral ROI $(949.6 \pm 48.1 \mathrm{~ms}$, $\mathrm{p}<0.005)$. The regional differences were similar at $3.0 \mathrm{~T}$ (Table 1).

Conclusion T1 values vary according to myocardial location. The explanation may be related to myocardial structure/function relationships and merits further study.

Abstract 27 Table 1 Segmental myocardial T1 times (mean \pm SD, ms)

\begin{tabular}{lll}
\hline & $1.5 \mathrm{~T}$ & $3.0 \mathrm{~T}$ \\
\hline Basal slice & & \\
Anterior & $940.6 \pm 56.5$ & $1132.5 \pm 43.3$ \\
Anterioseptal & $958.9 \pm 34.6$ & $1152.0 \pm 33.6$ \\
Inferioseptal & $964.5 \pm 32.9$ & $1150.8 \pm 30.7$ \\
Inferior & $963.6 \pm 42.6$ & $1151.1 \pm 40.2$ \\
Inferiolateral & $969.8 \pm 45.8$ & $1139.3 \pm 42.8$ \\
Anteriolateral & $931.7 \pm 46.7$ & $1133.4 \pm 42.1$ \\
Mid-ventricular slice & & \\
Anterior & $921.9 \pm 49.3$ & $1136.5 \pm 80.2$ \\
Anterioseptal & $944.1 \pm 50.0$ & $1154.6 \pm 51.2$ \\
Inferioseptal & $957.3 \pm 39.1$ & $1154.3 \pm 45.7$ \\
Inferior & $943.8 \pm 47.2$ & $1139.4 \pm 54.0$ \\
Inferiolateral & $945.1 \pm 58.1$ & $1149.9 \pm 69.0$ \\
Anteriolateral & $915.8 \pm 57.1$ & $1136.5 \pm 80.2$ \\
Apical slice & & \\
Anterior & $934.5 \pm 42.2$ & $1176.2 \pm 62.2$ \\
Septal & $962.9 \pm 47.7$ & $1185.3 \pm 55.4$ \\
Inferior & $943.0 \pm 51.7$ & $1156.0 \pm 59.4$ \\
Lateral & $936.5 \pm 48.0$ & $1189.8 \pm 53.0$ \\
\hline & &
\end{tabular}

